ISSN 0103-9954

\title{
RELAÇÃO ENTRE O ÍNDICE DE AVERMELHAMENTO DO SOLO E O ESTOQUE DE CARBONO NA BIOMASSA AÉREA DA VEGETAÇÃO DE CERRADO
}

\section{RELATIONSHIP BETWEEN REDNESS INDEX OF SOIL AND CARBON STOCK OF AERIAL BIOMASS IN CERRADO VEGETATION}

\author{
Otacilio Antunes Santana ${ }^{1}$ José Imaña Encinas ${ }^{2}$ Euzelina dos Santos Borges Inácio ${ }^{3}$ \\ Laerte Bezerra de Amorim ${ }^{4}$ João Luiz Jardim Vilaverde ${ }^{5}$
}

\begin{abstract}
RESUMO
A variação da cor do solo é uma resposta relacionada principalmente a sua composição física, química, biológica e mineralógica. O sistema de cores de Munsell é baseado na cor de forma objetiva, em que, através dela é possível avaliar diversos fatores do solo, como a presença de óxidos de ferro, granulometria e material orgânico. O objetivo do trabalho foi realizar uma relação e identificação entre o índice de avermelhamento (IAV) com o estoque de carbono presente na parte aérea da vegetação de Cerrado. O trabalho foi realizado em sete municípios do Oeste Baiano, em áreas nativas de Cerrado, nas fitofisionomias: Floresta de Galeria, Cerradão, Cerrado stricto sensu e Campo Sujo. A determinação da cor foi efetuada no campo em amostras de solo úmido, empregando-se a caderneta de Munsell. A partir da avaliação da matiz, valor e croma, foi calculado o índice de avermelhamento (IAV). A estimativa do estoque de carbono acima do solo foi realizada a partir dos compartimentos: tronco e galhos, tanto das árvores vivas quanto árvores mortas, através de uma equação alométrica desenvolvida para o Cerrado. $\mathrm{O}$ índice de avermelhamento da cor do solo na camada de 0,00 a $0,20 \mathrm{~m}$ possui uma relação diretamente proporcional e significativa com o estoque de carbono da biomassa aérea da vegetação de Cerrado, podendo ser extrapolado o cálculo do estoque de carbono (EC) pelo valor do índice de avermelhamento (IAV) do solo pela equação [EC $=0,4936 \cdot$ IAV + $4,2286(-1,5 \%)]$. A praticidade pela coleta dos dados de IAV, através da carta de Munsell e a confiabilidade do ajuste, dado pelo erro residual menor do que 5\%, faz com que esta relação possa ser utilizada para fins acadêmicos e coorporativos em estimação dos estoques de carbono para aplicação em manejos florestais e ambientais. A variação de EC entre as fitofisionomias estudadas faz-se, principalmente, pela distinta densidade de indivíduos arbóreos por hectare. A variação do IAV nas áreas estudadas é devido à matéria orgânica acumulada na superfície do solo, ao conteúdo mineral e à capacidade de drenagem, segundo inferências da literatura, refletindo assim nas distintas cores do solo nas fitofisionomias estudadas.
\end{abstract}

Palavras-chave: cor do solo; fitofisionomia; teor de ferro, carta de cores de Munsell.

\footnotetext{
ABSTRACT

The color variety of soil is response of its physical, chemical, biological and mineralogical composition. The Munsell color system is based on an objective color evaluation in which it is possible to evaluate different

1 Biólogo, Dr., Professor Adjunto do Departamento de Biofísica e Radiobiologia, Centro de Ciências Biológicas, Universidade Federal de Pernambuco, Av. Professor Moraes Rego, s/n, Cidade Universitária, CEP 50670-901, Recife (PE), Brasil. otacilio.santana@ufpe.br

2 Engenheiro Florestal, Ph.D., Professor Titular do Departamento de Engenharia Florestal, Universidade de Brasília, Caixa Postal 04357, CEP 70910-900, Brasília (DF), Brasil. imana@unb.br

3 Engenheira Agrônoma, Dra., Professora Adjunta do Departamento de Biofísica e Radiobiologia, Centro de Ciências Biológicas, Universidade Federal de Pernambuco, Av. Professor Moraes Rego, s/n, Cidade Universitária, CEP 50670-901, Recife (PE), Brasil. euzelina.inacio@ufpe.br

4 Engenheiro Agrônomo, Msc., Doutorando pela Universidade Federal de Pernambuco, Centro de Tecnologia, Departamento de Energia Nuclear, Av. Prof. Luiz Freire, 1000, Cidade Universitária, CEP 50740-540, Recife (PE), Brasil. laerteamorim@yahoo.com.br

5 Biólogo, Graduando pela Universidade Federal de Pernambuco, Centro de Ciências Biológicas, Ciências Biológicas - Bacharelado, Av. Professor Moraes Rego, s/n, Cidade Universitária, CEP 50670-901, Recife (PE), Brasil.joaov.jardim@gmail.com

Recebido para publicação em 14/02/2012 e aceito em 16/08/2012
} 
color soil factors such as the presence of iron oxides, particle size, and accumulated organic material. The objective of this study was to identify the relation between the redness index (IAV) of soil and the amount of carbon content present in the aerial part of Cerrado vegetation. The study was conducted in seven cities in western Bahia state, in native areas of Cerrado. The determination o soil color was made in the field, with moist soil samples, using the Munsell color chart. From the evaluation of hue, value and chroma, the redness index (IAV) was calculated. The estimation of carbon stock above ground was carried out from: trunk and branches, both living and dead trees by the allometric equation developed in Cerrado areas. The redness index of soil, from the layer 0.00 to $0.20 \mathrm{~m}$, has a significant and directly proportional relationship with the carbon stock of above ground biomass of Cerrado vegetation. This may be extrapolated to calculate the carbon stock (EC) by the redness index (IAV) of soil by the equation [EC $=0.4936 \cdot \mathrm{IAV}+4.2286$ $(-1.5 \%)]$. The practicality of sampling of data by IAV with Munsell chart, and the reliability of data fit by results of residual error less than 5\%, make that this relationship may be used for academic and corporative in carbon stocks estimation for use in forest and environmental management. The EC variation between the studied physiognomies is mainly by distinct density of tree individuals per hectare. The variation of IAV in studied areas is due to the accumulated organic matter on the soil surface, the mineral content and drainage capacity, according to inferences of literature, and this reflecting the different colors of soil in the studied phytophysiognomies.

Keywords: soil color; phytophysiognomies; iron content, Munsell color chart.

\section{INTRODUÇÃO}

Os processos de formação do solo envolvem todos os processos físicos, químicos e biológicos gerados a partir da rocha matriz em conjunto com a matéria orgânica advinda da parte superior da superfície do solo pela deposição de materiais abióticos e bióticos (fauna e flora) (DOUGLASS; BOCKHEIM, 2006). De acordo com o tamanho das partículas constituintes do solo, este pode ser muito compacto, com partículas pequenas (argiloso), sendo assim menos permeável (excetuando os solos microagregados); ou até mesmo formado por grandes partículas (arenoso) e bem permeável (GRAHAM; O'GEEN, 2010). Além da granulometria, outro fator importante para a classificação dos solos é a quantidade de matéria orgânica relacionada a este, que pode variar enormemente entre zonas devido à quantidade depositada (CHIG et al., 2008).

Associado a estas variações texturais, o conteúdo da matéria orgânica nos solos dá uma característica visual distinta, geralmente mais escura (DOUGLASS; BOCKHEIM, 2006). A cor é vista como uma análise subjetiva de comparação, em que haveria uma imprecisão em comparar pequenas alterações dentro de uma curta variação amostral (LYNN; PEARSON, 2000). O sistema de cores de Munsell passou a atribuir um aspecto objetivo para a cor do solo, em um sistema empregado dentro da pedologia para a identificação de solos (MUNSELL, 1905).

Devido à importância da cor do solo, várias pesquisas têm sido realizadas nesta área do conhecimento, principalmente comparando o conteúdo de nutrientes com a cor do solo. Como o exemplo já mencionado, a cor do solo tende a ficar mais escura à medida que a concentração de matéria orgânica aumenta gerando uma proporcionalidade possível de ser calculada, onde esses solos escuros podem conter uma grande atividade microbiana e fertilidade (KETTERINGS; BIGHAM, 2000). Dentro de uma mesma área em que o solo é formado pelo mesmo material de origem mineral, interpreta-se que as áreas mais escuras são as de maior fertilidade, tornando importante, assim, a cor do solo como fator de comparação e necessária na classificação e relação com a vegetação (LYNN; PEARSON, 2000).

O parâmetro mais comum para o calculo da cor de um solo é dado pelo índice de avermelhamento (IAV), esse parâmetro pode ser influenciado também pela concentração mineral, por exemplo: Goetita (FeOOH - 1-2 $\mu \mathrm{m}$ ) 10YR 8/6Munsell amarelo, Hematita $\left(\mathrm{Fe}_{2} \mathrm{O}_{3} \sim 0,4 \mu \mathrm{m}\right) 5 \mathrm{R} 3 / 6 \mathrm{Munsel}$ vermelho, Ferri-hidrita $\left[\mathrm{Fe}(\mathrm{OH})_{3}\right]$ 2,5YR 3/6Munsell vermelho-escuro, Calcita $\left(\mathrm{CaCO}_{3}\right)$ 10YR 8/2Munsell branco, Quartzo $\left(\mathrm{SiO}_{2}\right)$ 10YR 6/1Munsell cinza 
(LYNN; PEARSON, 2000), influindo diretamente nas propriedades do solo (ROSSEL et al., 2008). Este índice é baseado no sistema de cor de Munsell e utilizado por $90 \%$ dos pedólogos, permitindo a representação precisa de diversos fatores (BIGHAM; CIOLKOSZ; LUXMOORE, 1993), como apresentados na Tabela 1 .

O Cerrado é o segundo maior bioma brasileiro, superado somente pela Floresta Amazônica. Por sua grande variação de solo e de fitofisionomia, o Cerrado é um mosaico vegetacional. As fitofisionomias do Cerrado lato sensu estão situadas nos interflúvios (Cerrado stricto sensu, Cerradão, Campo Sujo, e outros) ou nas vias fluviais (Floresta de Galeria, Mata Ciliar e outras), criando distintas formações do solo, tanto pelos atributos geomorfológicos, posição hidrográfica, como também pela deposição de materiais advindos de seres vivos (EITEN, 2001). A relação solo e fitofisionomia é significativa neste bioma, resultado dos processos mencionados, tendo em vista, por exemplo, que fitofisionomias como Cerradão estão geralmente sob Neossolos e
Campo Sujo sob Latossolos (RUGGIERO et al., 2006).

Por ser um dos maiores biomas brasileiro é esperado que o Cerrado tenha uma grande biomassa aérea e um representativo estoque de carbono, ou seja, a quantidade de carbono contido em toda biomassa viva e morta e na parte decomposta, e sua capacidade de acúmulo (REZENDE et al., 2006). A biomassa e o estoque de carbono do ecossistema em questão são de fundamental importância para o equilíbrio dinâmico da região. A crescente mudança do uso da terra e, consequentemente, o desmatamento do Cerrado, transfere conteúdos de carbono da vegetação para a atmosfera, gerando um desequilíbrio nestes estoques no solo, modificando suas propriedades físicas e visuais (SCHWERTMANN, 1993). Assim sendo, formas de estimar a biomassa e o estoque de carbono local são fundamentais para o manejo da região, podendo ser aferidas as necessidades pontuais desta vegetação.

A hipótese deste trabalho foi que o índice de avermelhamento do solo possui uma relação di-

TABELA 1: Aplicação do índice de avermelhamento em algumas pesquisas.

TABLE 1: Redness index application in some researches.

\begin{tabular}{|c|c|}
\hline Trabalho & Área de Aplicação \\
\hline BARTHOLOMEUS; EPEMA; SCHAEPMAN (2007) & Relação do teor de ferro nas plantas \\
\hline BURKHARD; MÜLLER; LILL (2008) & Delimitação de ecótonos \\
\hline CAMPOS; DEMATTE (2004) & Discussão na classificação dos solos \\
\hline CAMPOS; DEMATTÊ; QUARTAROLI (2003) & Novas técnicas para a quantificação do teor de hematita \\
\hline DELGADO; AGUILAR; DELGADO (1994) & Micromorfologia \\
\hline DOUGLASS; BOCKHEIM (2006) & Pedogênese \\
\hline GALVÃO; VITORELLO; FORMAGGIO (1997) & Diferenciação de horizontes \\
\hline HOUSSA; PION; YÉSOU (1996) & Mineralogia \\
\hline HOWARD; CLAWSON; DANIELS (2012) & Cronossequência do solo \\
\hline KETTERINGS; BIGHAM (2000) & Fertilidade do solo \\
\hline KUMARAVEL et al. (2010) & Suscetibilidade magnética \\
\hline LEVIN et al. (2007) & Movimentação de dunas \\
\hline MADEIRA NETTO; ROBBEZ-MASSON; MARTINS (2006) & Delimitação das classes de solos \\
\hline MATHIEU et al. (1998) & Cartografia da degradação do solo \\
\hline MULDER et al. (2011) & Sensoriamento remoto e geoprocessamento \\
\hline PARK et al. (2010) & Relação com a sensibilidade ambiental de espécies \\
\hline RHOTON; SJOERD (2008) & Erodibilidade do solo \\
\hline ROSKIN et al. (2012) & Efeitos eólicos no solo \\
\hline ROSSEL et al. (2006) & Identificação da textura \\
\hline ROSSEL; FOUAD; WALTER (2008) & Presença e quantificação de carbono orgânico \\
\hline TEREFE et al. (2008) & Resposta ao aumento de temperatura \\
\hline
\end{tabular}


reta com o estoque de carbono da biomassa aérea da vegetação de Cerrado. Dessa forma, o objetivo geral deste trabalho foi sistematizar, através de uma possível relação significativa entre a cor do solo, mensurada pelo IAV e o estoque de carbono da biomassa aérea de algumas fitofisionomias de Cerrado, um método para estimação deste estoque através da mensuração do IAV dado pelas variáveis independentes obtidas a partir da carta de cores de Munsell, de modo a ser utilizado como ferramenta eficiente para o levantamento estimado do estoque de carbono, importante na demanda de praticidade coorporativa e acadêmica.

\section{MATERIAIS E MÉTODOS}

Aárea de estudo abrangeu sete municípios do Oeste Baiano: Riachão das Neves $\left(5.840,191 \mathrm{~km}^{2}\right)$, Luís Eduardo Magalhães $\left(4.018,778 \mathrm{~km}^{2}\right)$, Barreiras $\left(7.895,241 \mathrm{~km}^{2}\right)$, São Desidério (14.819, $\left.585 \mathrm{~km}^{2}\right)$, Correntina $\left(12.142,427 \mathrm{~km}^{2}\right)$, Jaborandi $\left(9.479,853 \mathrm{~km}^{2}\right)$ e Cocos $\left(10.0854,274 \mathrm{~km}^{2}\right)$, localizados no estado da Bahia e à margem esquerda do Rio São Francisco (Figura 1), com extensão total de $64.280,349 \mathrm{~km}^{2}$, banhados pelas Bacias dos rios Grande, Preto, Corrente, Pratudão e Pratudinho e Carinhanha. Nesta região houve uma perda de área

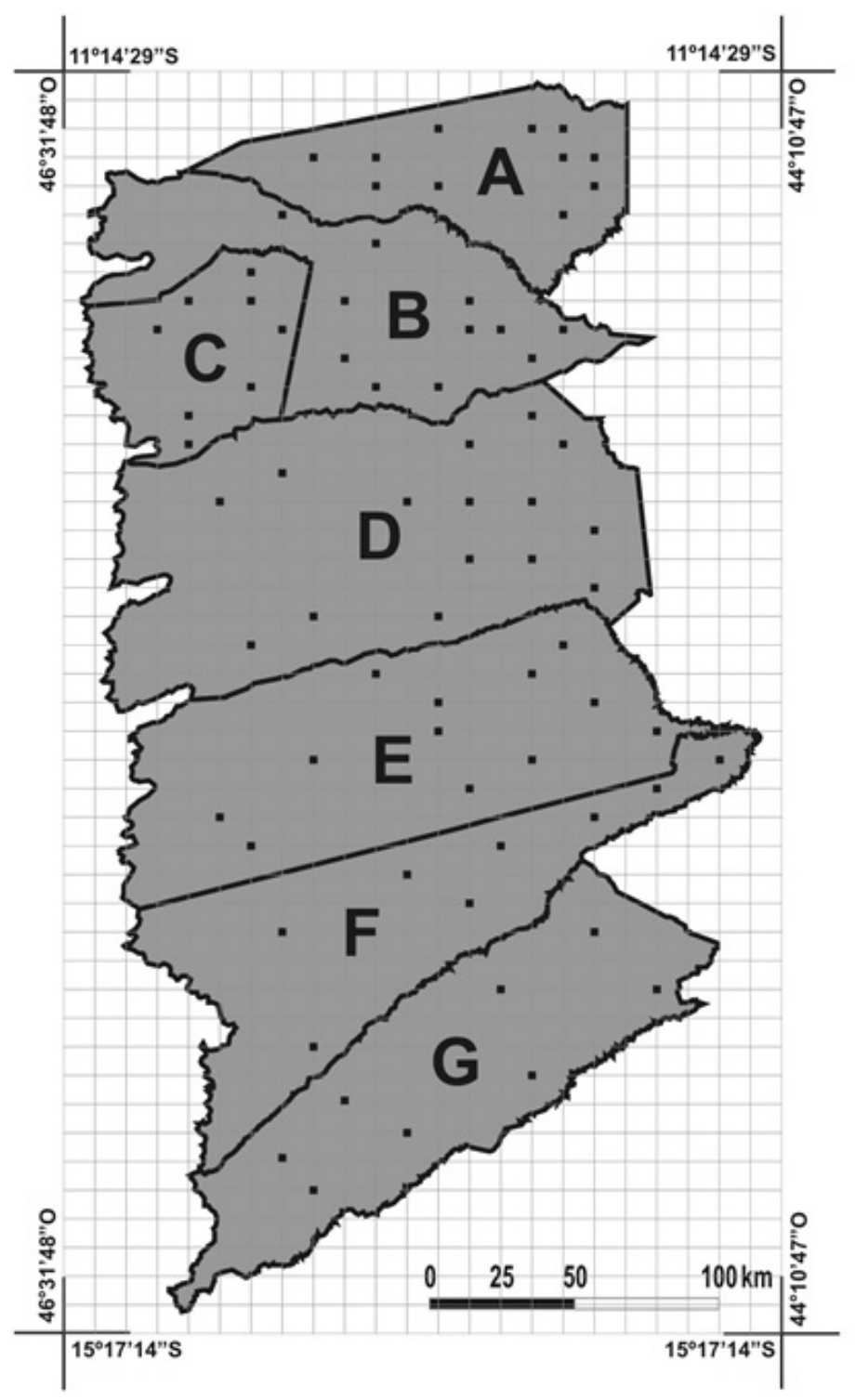

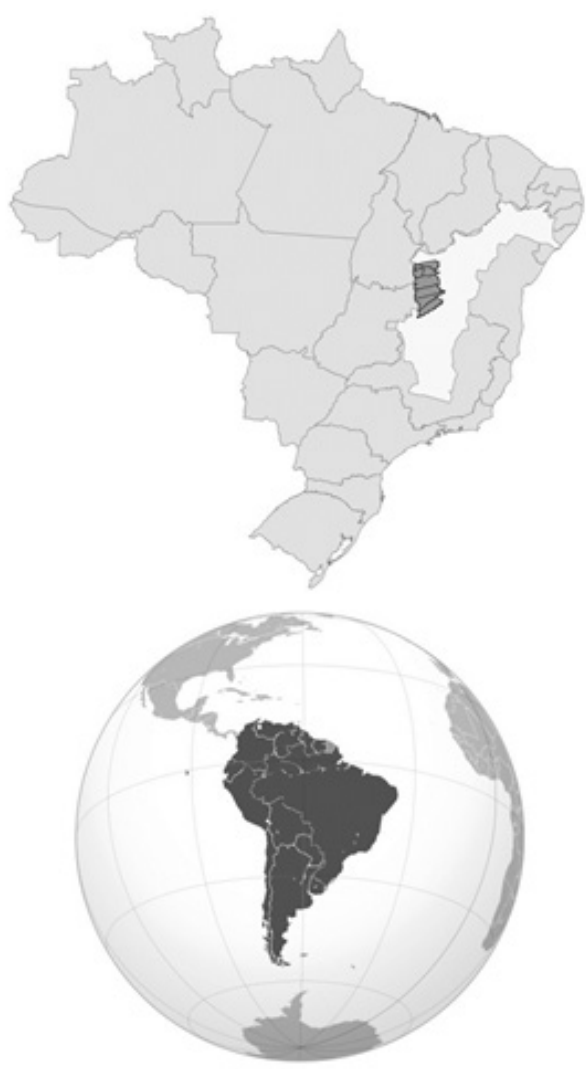

América do Sul

Brasil

Bacia do Rio São Francisco

Oeste Baiano

Municipios: A) Riachão das Neves;

B) Barreiras; C) Luís Eduardo Magalhães;

D) São Desidério; E) Correntina;

F) Jaborandi e G) Cocos.

- Pontos de amostragem dos dados

FIGURA 1: Localização da área de estudo apresentando os sete municípios estudados no Oeste Baiano, Bahia, Brasil.

FIGURE 1: Localization of study areas presenting the seven studied cities in western Bahia state, Brazil. 
de Cerrado de $40 \%$ para agropecuária e área urbana, entre o período de 1986 a 2002 (BRANNSTROM et al., 2008). O cerrado apresenta diversas fitofisionomias, entretanto, para a área em estudo foram encontrados os seguintes tipos: 1) Floresta de Galeria (floresta sempre-verde com árvores entre 20 e 30 metros e solos geralmente profundos e ricos em matéria orgânica na camada superficial); 2) Cerradão (floresta que apresenta elementos xeromórficos com a altura das árvores entre 8 e $15 \mathrm{~m}$ e predominância de Latossolo Vermelho); 3) Cerrado stricto sensu (fitofisionomia característica do bioma Cerrado com árvores baixas, entre 7 e 15 metros, e ampla variação de tipos de solo); e 4) Campo Sujo (fisionomia herbáceo-arbustiva com arbustos e subarbustos espaçados entre si, os solos variam de rasos a pouco profundos).

As precipitações se apresentam superiores a $1000 \mathrm{~mm}$ anuais e a evapotranspiração é de 1400 a $1600 \mathrm{~mm}$ anuais. O período chuvoso situa-se entre outubro e março e o período seco, com déficit hídrico, de abril a setembro. O veranico, que ocorre geralmente no mês de janeiro, é classificado como pequeno e o menos frequente entre os cerrados nordestinos. A temperatura média é de $22^{\circ} \mathrm{C}$ e o clima é classificado com tropical, sendo Aw de KöppenGeiger (IBGE, 2009).

Quanto à geologia, a área de estudo está situada na cobertura sedimentar de arenito formada no Cretáceo, sendo a unidade de relevo denominado de Chapadão Ocidental do São Francisco, a uma altitude que varia aproximadamente de 300 a $700 \mathrm{~m}$. Os solos são representados principalmente pelos Latossolos, podendo ocorrer Cambissolos, Neossolos, Plintossolos e Gleissolos, e se formam sob rochas sedimentares (Calcário, Travertino, Dolomita e Arenito). Em termos de potencialidade agrícola, os solos são classificados como boa e boa a regular, respectivamente, sem limitações e com poucas deficiências de nutrientes (IBGE, 2009).

Em cada intersecção dos quadrantes de 10 x $10 \mathrm{~km}$ que ocorria vegetação nativa, conforme visualizado na Figura 1, foram estabelecidas parcelas de $100 \times 100 \mathrm{~m}$, onde foram coletadas as amostras de solo e os dados de vegetação, em um total de 73 pontos amostrados (Figura 1 ).

A coleta do solo $(0,00$ a $0,20 \mathrm{~m})$ foi realizada em cada parcela, com o auxílio de um trado, em 10 pontos aleatórios dentro da parcela. Para a comparação com os dados de vegetação foi tomada a média dos 10 pontos para os valores determinados para a cor, conforme descrito posteriormente.

A determinação da cor foi efetuada no campo em amostras de solo úmido, empregando-se a carta de Munsell. A partir da avaliação de matiz, valor e croma, foi calculado o índice de avermelhamento (IAV) (TORRENT; BARRÒN, 1993):

$$
I A V=\frac{(10-M) \times C}{V}
$$

em que, $M=$ matiz $(7.5 \mathrm{R}=0 ; 10 \mathrm{R}=1$; $2.5 \mathrm{YR}=2 ; 5 \mathrm{YR}=3 ; 7.5 \mathrm{YR}=4 ; 10 \mathrm{YR}=5 ; 25 \mathrm{YR}$ $=6 ; 2.5 \mathrm{Y}=7) ; V=$ valor; $C=$ croma.

A estimativa do estoque de carbono da parte aérea da vegetação foi realizada a partir dos compartimentos: tronco e galhos, tanto nas árvores vivas quanto nas mortas (PAIVA; REZENDE; PEREIRA, 2011), por meio da equação alométrica desenvolvida para o Bioma Cerrado (REZENDE et al., 2006):

$$
E C=-0,24564+0,01456 \times D b^{2} \times H \quad(\text { Eq. } 2)
$$

Em que: $E C=$ estoque de carbono em $\mathrm{kg}$ por árvore; $D b=$ diâmetro da base, tomado a $0,30 \mathrm{~m}$ do solo $(\mathrm{cm}) ; H=$ altura total da árvore $(\mathrm{m})$. Posteriormente, foi calculado o ECtotal por parcela de amostragem $(100 \times 100 \mathrm{~m})$ :

$$
E C \text { total }=E C \times n \quad \text { (Eq. 3) }
$$

Em que: $E C$ total $=$ estoque de carbono total por parcela, transformados na unidade $\mathrm{tha}^{-1} ; \mathrm{n}=$ número total de árvores por parcelas.

Foram considerados todos os indivíduos lenhosos com diâmetro da base, tomado a $0,30 \mathrm{~m}$ do solo, igual ou superior a $5 \mathrm{~cm}$. Galhos e seções com diâmetro menor que $3 \mathrm{~cm}$ foram desconsiderados neste modelo. Na estimativa do estoque de carbono, este modelo considera um conteúdo de carbono correspondente a $50 \%$ da biomassa seca (VALE; FELFILI, 2005). A classificação da fitofisionomia foi realizada de acordo com Eiten (2001), pela densidade de árvores por hectare, presença de curso d'água próximo e altura das árvores (Floresta de Galeria $>500$ ind. ha ${ }^{-1}$ com curso d'água próximo e altura média das árvores $>15 \mathrm{~m}$; Cerradão $>500$ ind. ha $^{-1}$ sem curso d'água próximo e altura média das árvores $>12 \mathrm{~m}$; Cerrado stricto sensu 400 $<$ ind. ha ${ }^{-1}>200$, altura média das árvores $<12 \mathrm{~m}$, e Campo Sujo $<50$ árvores por hectare) (Figura 2). 

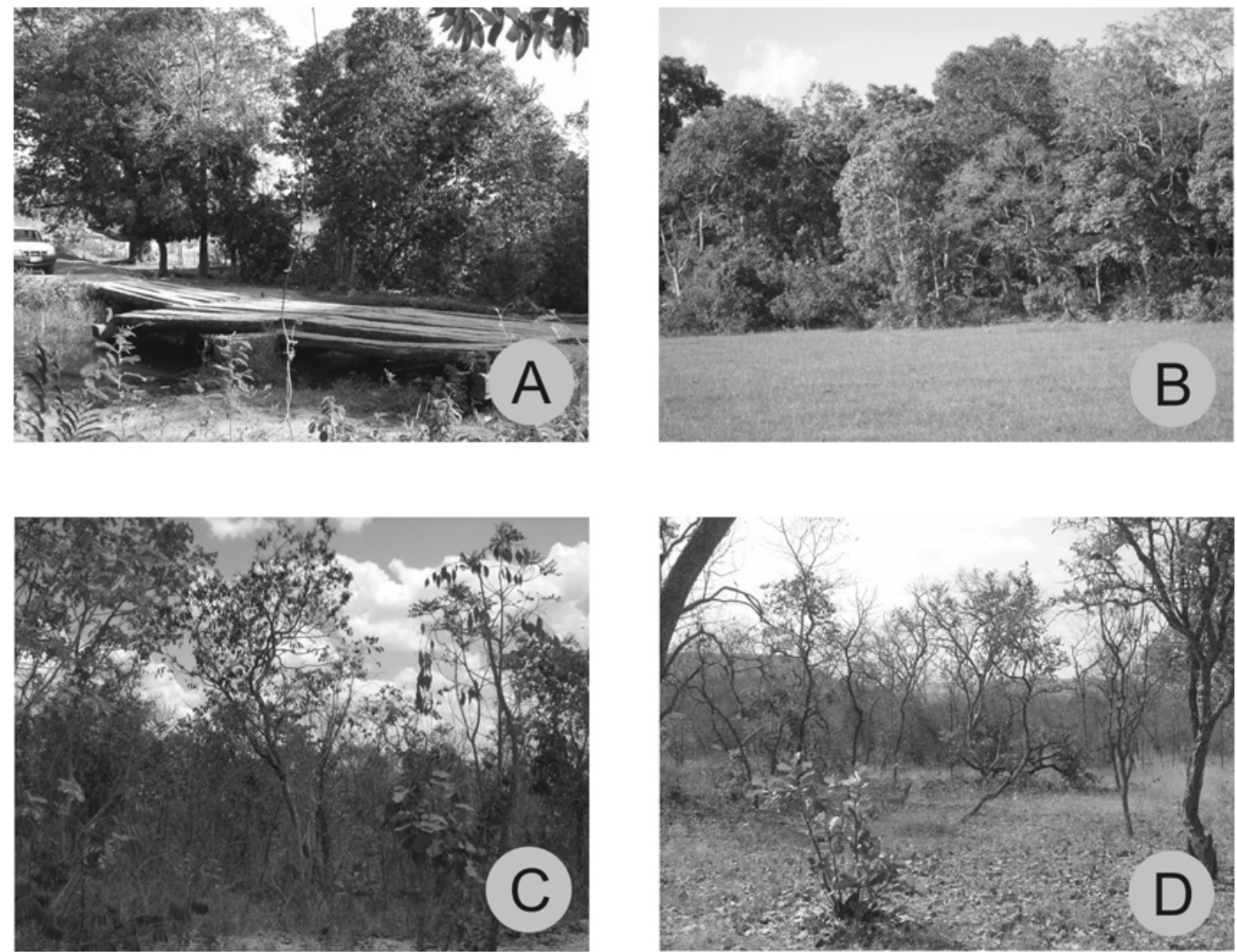

FIGURA 2: Fitofisionomias estudadas: A) Floresta de Galeria; B) Cerradão; C) Cerrado stricto sensu e D) Campo Sujo.

FIGURE 2: Studied phytophysiognomies: A) Floresta de Galeria; B) Cerradão; C) Cerrado stricto sensu and D) Campo sujo.

O teste de hipótese não paramétrico MannWhitnney foi utilizado para obter o nível de significância (p) entre as fitofisionomias nas variáveis EC e IAV. Análises de correlação de Pearson foram efetuadas entre as variáveis EC, IAV com a quantidade de indivíduos por hectares. A análise de regressão foi realizada entre as variáveis EC e IAV para se obter a relação proporcional entre as variáveis através dos parâmetros significativos: $\mathrm{R}^{2}>0,5$; Erro $<0,05$; $\mathrm{p}<0,05$ e equação (ZAR, 2010).

Para a calibração do dado estimado pela equação encontrada através da análise de regressão, foi calculado o erro residual (ER), segundo a equação 4:

$$
E R(\%)=\frac{\text { real }- \text { estimado }}{\sum \text { real }- \text { estimado }} \times 100 \quad \text { (Eq. 4) }
$$

Em que, real é o dado amostrado e o estima$d o$, o dado gerado a partir da equação.

\section{RESULTADOS E DISCUSSÃO}

Os valores referentes ao estoque de carbono na vegetação e ao índice de avermelhamento foram maiores em regiões onde há a presença de vegetação mais densa (Tabela 2), como na Floresta de Galeria e o Cerradão; enquanto valores menores foram encontrados tanto no Cerrado stricto sensu como no Campo Sujo, dados que sugerem uma correlação com a densidade da vegetação local. Dados semelhantes foram encontrados também por pesquisadores que relacionaram o EC, com a densidade vegetal, tanto em Cerrado (REZENDE et al., 2006) como na Amazônia (CHIG, 2008).

Comparando-se os resultados do estoque de carbono entre as áreas fitofisionômicas do Cerrado, os resultados foram significativos $(p<0,001)$, ou seja, mostraram que as fitofisionomias estudadas comportam-se de maneira distinta no acúmulo de carbono na parte aérea da vegetação (Tabela 2). E 
TABELA 2: Estoque médio de carbono (EC) da parte aérea da vegetação nativa, média e desvio padrão do índice de avermelhamento do solo (IAV), fitofisionomias de Cerrado e densidade arbórea (indivíduos por hectare (ind. ha $^{-1}$ ) com diâmetros da base $>5 \mathrm{~cm}$ ).

TABLE 2: Mean of carbon stocks (EC) in aerial tree part of native vegetation, mean and standard deviation of redness index of soil (IAV) in phytophysiognomies of Cerrado and tree density (Individuals per hectares (ind. ha ${ }^{-1}$ ) with diameter of ground $>5 \mathrm{~cm}$.

\begin{tabular}{|c|c|c|c|c|}
\hline \multirow{2}{*}{ Fitofisionomia } & \multirow{2}{*}{$\begin{array}{c}\mathrm{EC} \\
\left(\mathrm{t} \mathrm{ha}^{-1}\right)^{1}\end{array}$} & \multicolumn{2}{|c|}{$\mathrm{IAV}^{2}$} & \multirow{2}{*}{ ind. ha- } \\
\hline & & Média & \pm & \\
\hline $\begin{array}{c}\text { Floresta de Galeria } \\
\text { (FG) }\end{array}$ & 12.287 & 14.257 & 0,701 & 1.347 \\
\hline Cerradão (Cdão) & 11.831 & 12.965 & 0,450 & 922 \\
\hline $\begin{array}{l}\text { Cerrado stricto } \\
\text { sensu (Css) }\end{array}$ & 8.013 & 7.843 & 0,475 & 399 \\
\hline Campo Sujo (CS) & 4.223 & 1.438 & 0,452 & 47 \\
\hline
\end{tabular}

Em que: ${ }^{1} \mathrm{p}<0,001$ : FG vs Cdão; FG vs Css; FG vs CS; Cdão vs Css; Cdão vs CS; Css vs CS. ${ }^{2} \mathrm{p}<0,001$ : FG vs Css; FG vs CS; Cdão vs Css; Cdão vs CS; Css vs CS; p > 0,05 FG vs Cdão.

isto pode ser certificado pelo valor de $\rho(0,981)$ entre as variáveis EC e a quantidade de indivíduos por hectares, e motivado pelos diferentes volumes de madeira e índices de área foliar encontrados, quando analisadas diferentes fitofisionomias (SANTANA; CUNIAT; IMAÑA-ENCINAS, 2010; VIANNA et al., 2010; SANTANA et al., 2011).

Em relação ao índice de avermelhamento (IAV), as fitofisionomias também se mostraram distintas $(p<0,001)$, exceto quando comparados o Cerradão e a Floresta de Galeria $(p=0,145)$. Mas também mostrando uma correlação significativa com a variável quantidade de indivíduos por hectares $(\rho=0,903)$. Esses dados deram subsídios para a diferenciação inicialmente da cor do solo superficial, primeiramente motivada pelo distinto acúmulo de matéria orgânica entre as fitofisionomias advindos principalmente da parte aérea da vegetação e toda a fauna associada. Em solos situados abaixo de ambientes florestais, como a Floresta de Galeria e Cerradão, a cor do solo é geralmente escura devido a uma espessura maior de serapilheira que está em decomposição (SANTANA et al., 2010), juntamente com a presença de alguns organismos vivos e micro- -organismos aeróbicos, formando o húmus, de caraterística Munsell 10R 2/1 (BIGHAM; CIOLKOSZ; LUXMOORE, 1993). Neste húmus e na matéria orgânica, bactérias desempenham um papel importante, pois ajudam no processo de remoção e redução do ferro $\left(\mathrm{Fe}^{3+} \rightarrow \mathrm{Fe}^{2+}\right)$ e manganês $\left(\mathrm{Mn}^{4+} \rightarrow \mathrm{Mn}^{2+}\right)$, modificando a cor superficial do solo, pois são elementos essenciais para o IAV (SCHWERTMANN, 1993; LYNN; PEARSON, 2000). A presença de um curso d'água próximo à Floresta de Galeria foi também um importante fator para manutenção destes micro-organismos aeróbicos.

Estes elementos, ferro e manganês, são parte da característica pedomorfogeológicas que varia de região para região. Em ambientes de Floresta de Galeria e Cerradão são predominantemente formados sob Latossolos Vermelho e Vermelho-Amarelo (OLIVEIRA; MARQUIS, 2002; EITEN, 2001), com um maior conteúdo de minerais do tipo Hematita $\left(\mathrm{Fe}_{2} \mathrm{O}_{3}-10 \mathrm{R} 4 / 8 \mathrm{Munsell}\right)$ do que Goetita (FeOOH - 10YR 8/6Munsell) (BARBOSA; LACERDA; BILICH, 2009), que dão características avermelhadas; e para Cerrado stricto senso e Campo Sujo, formados sob solos Latossolos Vermelho-Amarelo, Latossolos Amarelos, Neossolos Quartzarênicos e Cambissolos (EITEN, 2001; OLIVEIRA; MARQUIS, 2002) a relação é o contrário, mais conteúdo de Goetita do que Hematita, que fornecem característica amarelada. Outro fator importante que influenciou o IAV foi a capacidade de drenagem do solo, visto que os solos com esta maior capacidade são os descritos para Cerrado stricto sensu e do Campo Sujo, o que caracterizam o maior conteúdo de Goetita (BARBOSA; LACERDA; BILICH, 2009), que possui um tamanho maior $(\sim 1-2 \mu \mathrm{m})$ do que a Hematita $(\sim 0,1-0,4 \mu \mathrm{m})$ que geralmente é drenada (SCHWERTMANN, 1993).

Relacionando-se o índice de avermelhamento do solo com o estoque de carbono da vegetação pôde-se verificar uma relação diretamente proporcional e significativa (Figura 3), indicando que, quanto maior o valor do IAV, maior o valor de EC na fitofisionomia estabelecida (Figuras 4 a 6). Para as áreas estudadas e conforme discutido, infere-se que a relação de causa e efeito se dá pela qualificação nesta relação do IAV como variável independente, pois esta variável reflete conteúdo de matéria orgânica e mineral presente, e a capacidade de drenagem do solo para áreas de Cerrado, propiciando distintos substratos para as espécies vegetais (BARBOSA; LACERDA; BILICH, 2009; PINHEIRO; DURIGAN, 2012). Estes substratos 


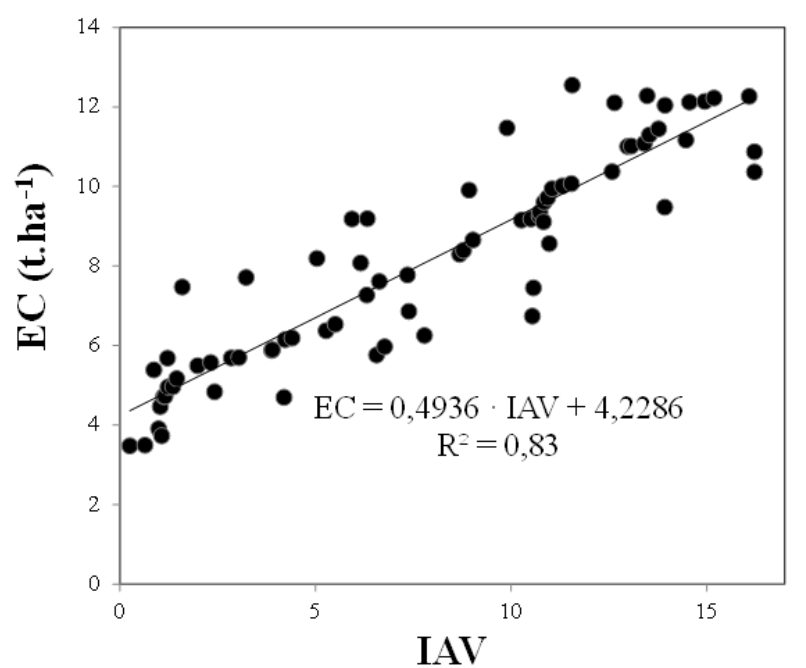

FIGURA 3: Relação entre o estoque de carbono (EC) na vegetação aérea de Cerrado e o índice de avermelhamento (IAV) do solo das áreas amostradas.

FIGURE 3: Relationship between the carbon stock (EC) in aerial part of Cerrado vegetation and the redness index (IAV) of soil of studied areas. são determinantes no estabelecimento das espécies vegetais e na dinâmica espacial destas espécies, ou seja, nos índices de densidade e frequência dos indivíduos vegetais para região do Cerrado (FELFILI et al., 2011). Outro índice da dinâmica populacional que é influenciado pelo substrato é o de dominância das espécies, calculado a partir da área basal dos indivíduos, influindo nas variáveis diâmetro e altura dos indivíduos arbóreos, que são variáveis diretas para o cálculo do estoque de carbono (REZENDE et al., 2006).

A estimação do estoque de carbono, como o realizado neste trabalho, é aplicável pela praticidade de mensuração via método aplicado com a carta de Munsell, estimação como esta, através de modelo com variáveis independentes são utilizadas em diversas empresas na área de manejo florestal e ambiental para redução do tempo de coletas de dados e, consequentemente, redução dos gastos com monitoramento e levantamentos finais (KÖHL et al., 2008; VIEIRA etal., 2008; WATERWORTH; RICHARDS, 2008; DJOMO; KNOHL; GRAVENHORST, 2011; ALVAREZ et al., 2012). E pela confiabilidade da
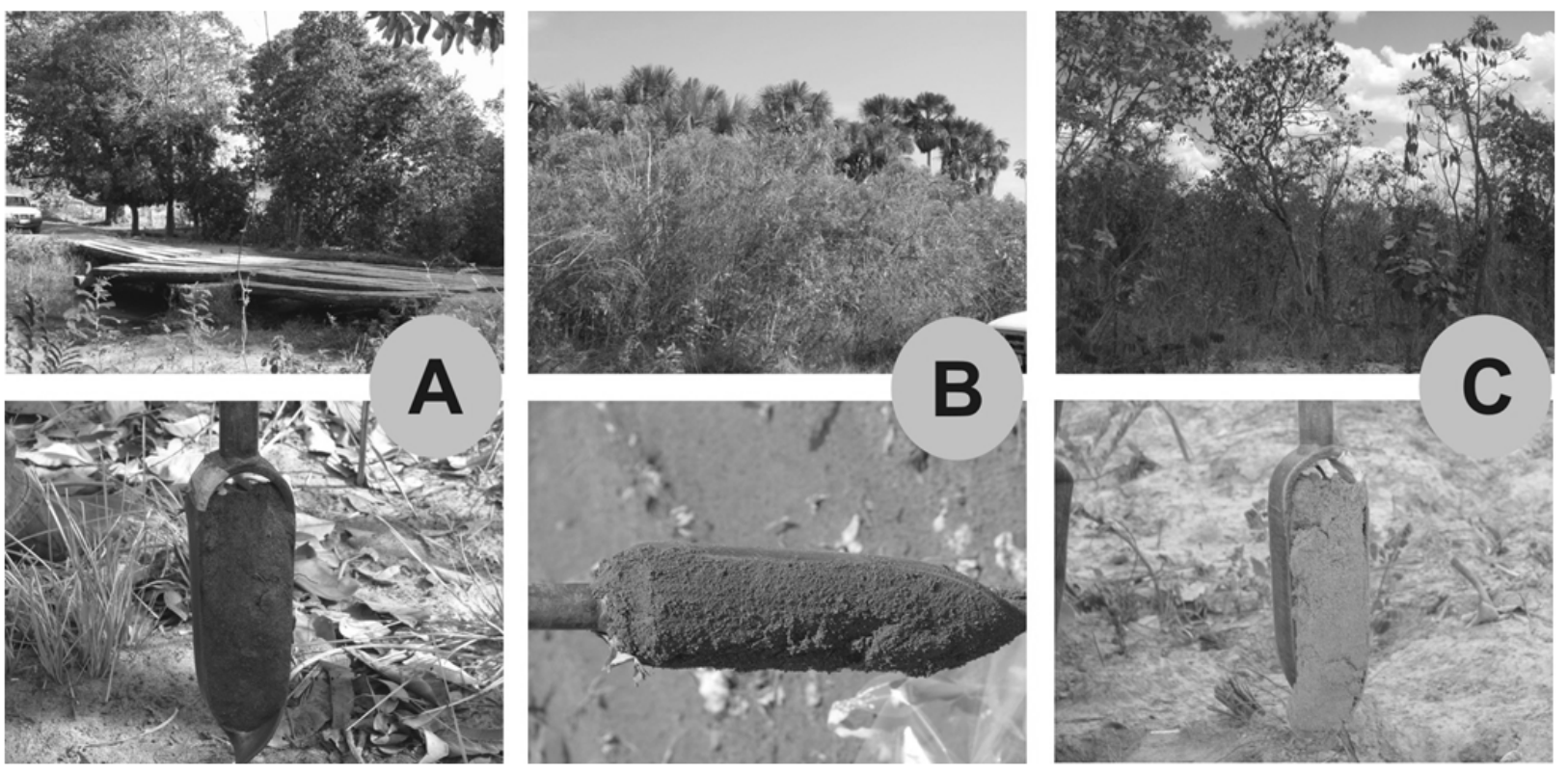

FIGURA 4: Matiz, valor (V) e croma (C) dos solos: A) 7.5R V5 C1 - Floresta de Galeria; B) 10R V5 C8 - Cerradão e C) 2.5YR V5 C8 - Cerrado stricto sensu.

FIGURE 4: Hue, value (V) and chroma (C) of soils: A) 7.5R V5 C1 - Floresta de Galeria; B) 10R V5 C8 - Cerradão and C) 2.5YR V5 C8 - Cerrado stricto sensu. 

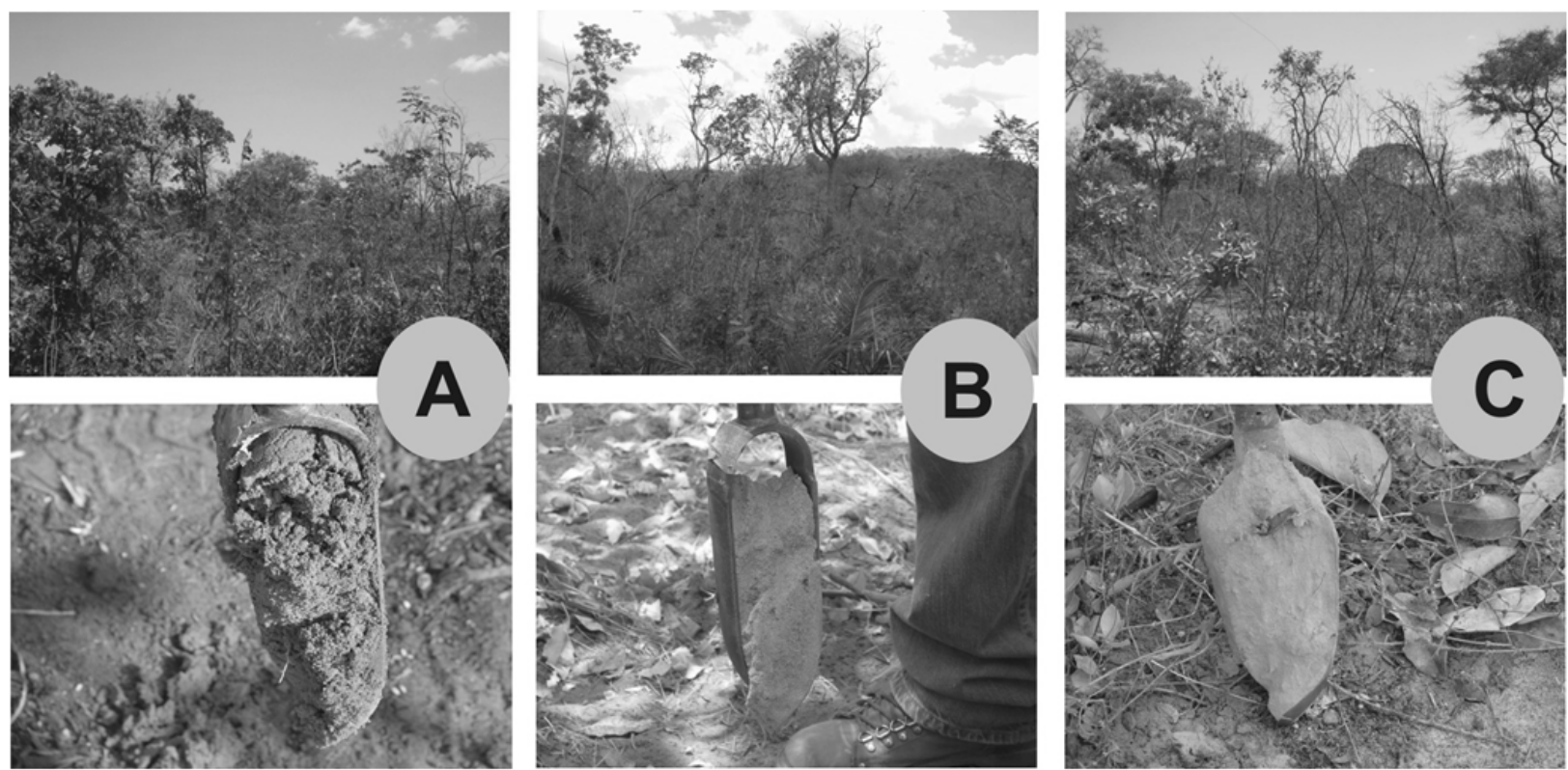

FIGURA 5: Matiz, valor (V) e croma (C) dos solos: A) 5YR V8 C8 - Cerradão; B) 5YR V6 C8 - Cerrado stricto sensu; e C) 7.5YR V7 C6 - Cerrado stricto sensu.

FIGURE 5: Hue, value (V) and chroma (C) of soils: A) 5YR V8 C8 - Cerradão; B) 5YR V6 C8 - Cerrado stricto sensu; and C) 7.5YR V7 C6 - Cerrado stricto sensu.
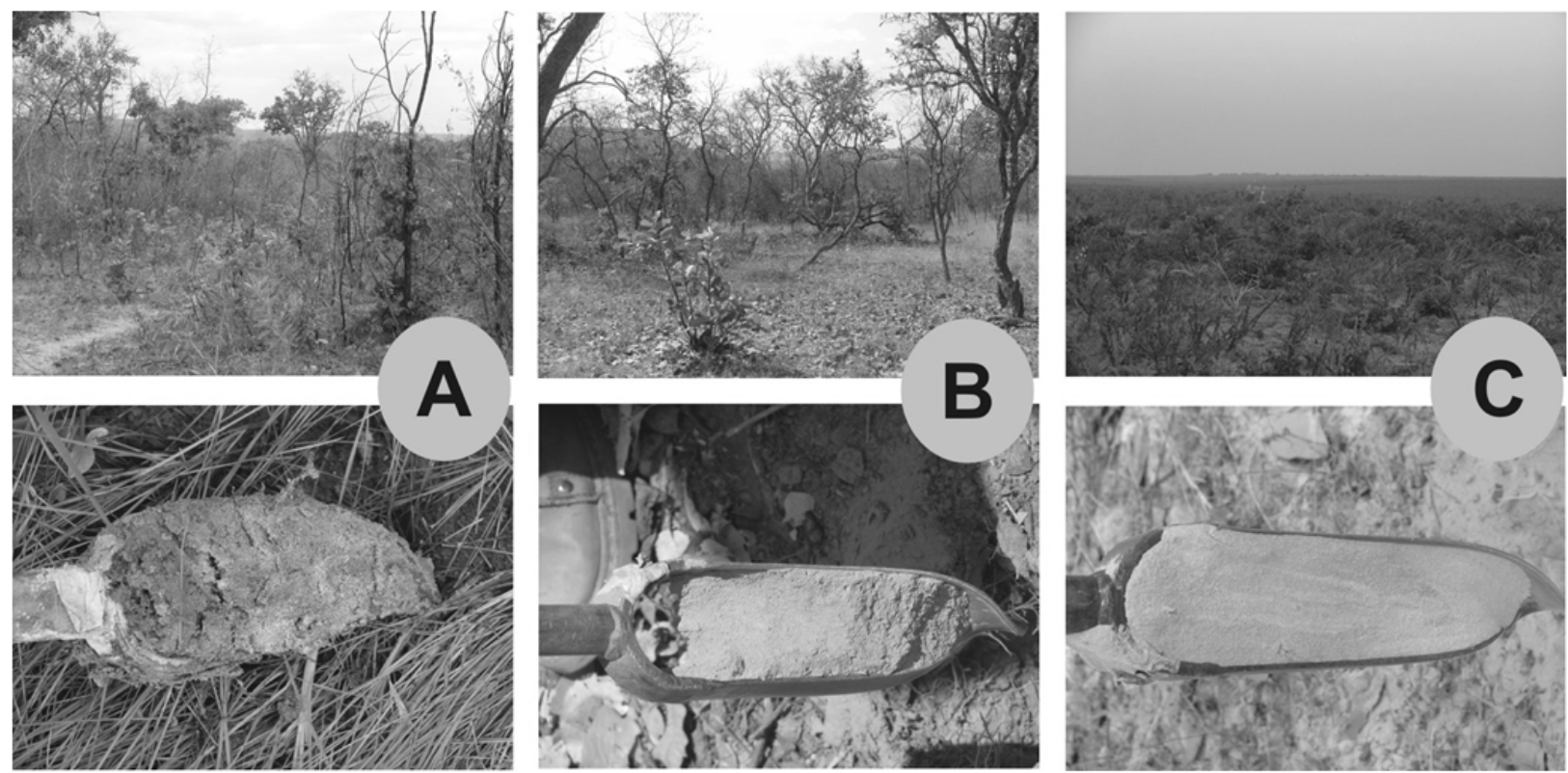

FIGURA 6: Matiz, valor (V) e croma (C) dos solos: A) 10YR V7 C4 - Cerrado stricto sensu; B) 25YR V8 C6 - Cerrado stricto sensu; e C) 7.5Y V8 C1 - Campo Sujo

FIGURE 6: Hue, value (V) and Chroma (C) of soils A) 10YR V7 C4 - Cerrado stricto sensu; B) 25YR V8 C6 - Cerrado stricto sensu; and C) 7.5Y V8 C1 - Campo Sujo. 
equação para estimação dos dados, visto o valor do erro residual dos dados menor do que $5 \%$, com uma superestimação de 1,5\% (Figura 7), resultados satisfatórios para o ajuste dos dados na análise de regressão e podendo ser agregada à equação a correção desta superestimação (COOK; WEISBERG, 1982), para calibração local dos dados $E C=0,4936$ $\cdot \operatorname{IAV}+4,2286(-1,5 \%)$.

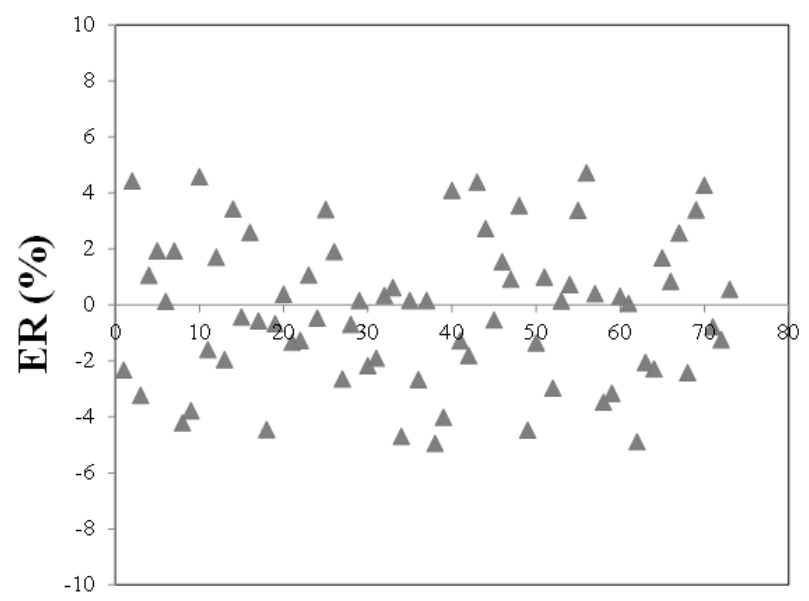

FIGURA 7: Erro residual da estimação do estoque de carbono pelo índice de avermelhamento do solo $(\mathrm{EC}=0,4936$ - IAV + 4,2286).

FIGURE 7: Residual error of carbon stock estimation by redness index of soil (EC $=0.4936 \cdot \mathrm{IAV}+4.2286)$.

\section{CONCLUSÕES}

O índice de avermelhamento da cor do solo na camada de 0,00 a $0,20 \mathrm{~m}$ possui uma relação diretamente proporcional e significativa com o estoque de carbono da biomassa aérea da vegetação de Cerrado, podendo ser extrapolado o cálculo do estoque de carbono (EC) pelo valor do índice de avermelhamento (IAV) do solo pela equação [EC = $0,4936 \cdot \mathrm{IAV}+4,2286(-1,5 \%)]$ gerada neste traba1ho. A praticidade pela coleta dos dados de IAV através da carta de Munsell e a confiabilidade do ajuste, dado pelo erro residual menor do que $5 \%$, faz com que esta relação possa ser utilizada para fins acadêmicos e coorporativos em estimação dos estoques de carbono para aplicação em manejos florestais e ambientais, conforme são utilizados diversos modelos de estimação de dados por empresas.

A variação de EC entre as fitofisionomias estudadas se faz principalmente pela distinta den- sidade de indivíduos arbóreos por hectare. A variação do IAV nas áreas estudadas é devido à matéria orgânica acumulada na superfície do solo, ao conteúdo mineral e à capacidade de drenagem, segundo inferências da literatura, refletindo assim nas distintas cores do solo nas fitofisionomias estudadas (Floresta de Galeria, Cerradão, Cerrado stricto sensu e Campo Sujo).

\section{AGRADECIMENTOS}

Ao Grupo de Pesquisa "Interface Biologia e Educação".

\section{REFERÊNCIAS BIBLIOGRÁFICAS}

ALVAREZ, E. et al. Tree above-ground biomass allometries for carbon stocks estimation in the natural forests of Colombia. Forest Ecology and Management, v. 267, n. 1, p. 297-308, 2012.

BARBOSA, I. O.; LACERDA, M. P. C.; BILICH, M. R. Relações pedomorfológicas nas Chapadas elevadas do Distrito Federal. Revista Brasileira de Ciências do Solo, v. 33, n. 5, p. 1373-1383, 2009.

BARTHOLOMEUS, H.; EPEMA, G.; SCHAEPMAN, M. Determining iron content in Mediterranean soils in partly vegetated areas, using spectral reflectance and imaging spectroscopy. International Journal of Applied Earth Observation and Geoinformation, v. 9, n. 2, p. 194-203, 2007.

BRANNSTROM, C. et al. Land change in the Brazilian Savanna (Cerrado), 1986-2002: Comparative analysis and implications for landuse policy. Land Use Policy, v. 25, p. 579-595. 2008.

BIGHAM, J. M.; CIOLKOSZ, E. J.; LUXMOORE, R. J. Soil Color. Madison: SSSA Special Publication. 1993. $172 \mathrm{p}$.

BURKHARD, B.; MÜLLER, F.; LILL, A. Ecosystem Health Indicators. Encyclopedia of Ecology, p. 1132-1138, 2008.

CAMPOS, R. C.; DEMATTE, J. A. M. Cor do solo: uma abordagem da forma convencional de obtenção em oposição à automatização do método para fins de classificação de solos. Revista Brasileira de Ciências do Solo, v. 28, n. 5, p. 853-863, 2004.

CAMPOS, R. C.; DEMATTÊ, J. A. M.; QUARTAROLI, C. F. Determinação indireta do teor de hematita na fração argila de solos a partir de dados de colorimetria e radiometria. Pesquisa Agropecuária Brasileira, v. 38, n. 4, p. 521-528, 
2003.

CHIG, L. A. et al. Distribuição espacial da granulometria, cor e carbono orgânico do solo ao longo de um transecto em microbacias na Amazônia meridional. Acta Amazonica, v. 38, p. 715-722, 2008.

COOK, R. D.; WEISBERG, S. Residuals and Influence in Regression. New York : Chapman and Hall, 1982. $230 \mathrm{p}$.

DELGADO, R.; AGUILAR, J.; DELGADO, G. Use of numerical estimators and multivariate analysis to characterize the genesis and pedogenic evolution of xeralfs from southern Spain. CATENA, v. 23, n. 3-4, p. 309-325, 1994.

DJOMO, A. N.; KNOHL, A.; GRAVENHORST, G. Estimations of total ecosystem carbon pools distribution and carbon biomass current annual increment of a moist tropical forest. Forest Ecology and Management, v. 261, n. 8, p. 1448-1459, 2011. DOUGLASS, D. C.; BOCKHEIM, J. G. Soilforming rates and processes on Quaternary moraines near Lago Buenos Aires, Argentina. Quaternary Research, v. 65, n. 2, p. 293-307, 2006.

EITEN, G. Vegetação natural do Distrito Federal. Brasília: SEBRAE. 162 p. 2001.

FELFILI, J. M. et al. Fitossociologia no Brasil: métodos e estudos de casos. Viçosa: Editora UFV, 2011.558 p. v. 1.

GALVÃO, L. S.; VITORELLO, I.; FORMAGGIO, A. R. Relationships of spectral reflectance and color among surface and subsurface horizons of tropical soil profiles. Remote Sensing of Environment, v. 61, n. 1, p. 24-33, 1997.

GRAHAM, R. C.; O'GEEN, A. T. Soil mineralogy trends in California landscapes. Geoderma, v. 154, n. 3-4, p. 418-437, 2010.

HOWARD, J. L.; CLAWSON, C. R.; DANIELS, W. L. A comparison of mineralogical techniques and potassium adsorption isotherm analysis for relative dating and correlation of Late Quaternary soil chronosequences. Geoderma, v. 179-180, p. 81-95, 2012.

HOUSSA, R.; PION, J. C.; YÉSOU, H. Effects of granulometric and mineralogical composition on spectral reflectance of soils in a Sahelian area.

ISPRS Journal of Photogrammetry and Remote Sensing, v. 51, n. 6, p. 284-298, 1996.

IBGE - Instituto Brasileiro de Geografia e Estatística. Mapas temáticos. Disponível em http://mapas. ibge.gov.br/ Acessado em 20.09.2009

KETTERINGS, Q. M.; BIGHAM J. M. Soil Color as an Indicator of Slash-and-Burn Fire Severity and
Soil Fertility in Sumatra, Indonesia. Soil Science Society of America Journal, v. 64, p. 1826-1833, 2000.

KÖHL, M. et al. Effect of the estimation of forest management and decay of dead woody material on the reliability of carbon stock and carbon stock changes-A simulation study. Forest Ecology and Management, v. 256, n. 3, 229-236, 2008.

KUMARAVEL, V. et al. Interrelation of magnetic susceptibility, soil color and elemental mobility in the Pliocene-Pleistocene Siwalik paleosol sequences of the NW Himalaya, India. Geoderma, v. 154, n. 3-4, p. 267-280, 2010.

LEVIN, N. et al. Dune whitening and inter-dune freshwater ponds in NE Brazil. Catena, v. 70, n. 1, p. 1-15, 2007.

LYNN, W. C.; PEARSON, M. J. The Color of Soil, exploring the chemistry of soil color. Science Teacher, v. 67, n. 5, p. 20-23, 2000.

MADEIRA NETTO, J.S.; ROBBEZ-MASSON, J. M.; MARTINS, E. Visible-NIR Hyperspectral Imagery for Discriminating Soil Types in the La Peyne Watershed (France). Developments in Soil Science, v. 31, p. 219-233, 2006.

MATHIEU, R. et al. Relationships between Satellite-Based Radiometric Indices Simulated Using Laboratory Reflectance Data and Typic Soil Color of an Arid Environment. Remote Sensing of Environment, v. 66, n. 1, p. 17-28, 1998.

MULDER, V. L. et al. The use of remote sensing in soil and terrain mapping - A review. Geoderma, v. 162, n. 1-2, p. 1-19, 2011.

MUNSELL, A. H. A color notation. Baltimore: G.H.Ellis Co, 1905. 89 p.

OLIVEIRA, P. S.; MARQUIS, R. J. The Cerrados of Brazil: Ecology and Natural History of a Neotropical Savanna. New York: Columbia University Press. 2002. 398 p.

PAIVA, A. O.; REZENDE, A. V.; PEREIRA, R. $\mathrm{S}$. Estoque de carbono em cerrado sensu stricto do Distrito Federal. Revista Árvore, v. 35, n.3, p. 527538. 2011.

PARK, A.; et al. Local and regional environmental variation influences the growth of tropical trees in selection trials in the Republic of Panama. Forest Ecology and Management, v. 260, n. 1, p. 12-21, 2010.

PINHEIRO, E. S.; DURIGAN, G. Diferenças florísticas e estruturais entre fitofisionomias do cerrado em Assis, SP, Brasil. Revista Árvore, v. 36, n. 1, p. 181-193, 2012.

REZENDE, A. V. et al. Comparação de modelos 
matemáticos para estimativa do volume, biomassa e estoque de carbono da vegetação lenhosa de um cerrado sensu stricto em Brasília, DF. Scientia Forestalis, v. 7 1, n. 2, p. 65- 73. 2006.

RHOTON F. E.; SJOERD, W. D. Erodibility of a soil drainage sequence in the loess uplands of Mississippi. Catena, v. 75, n. 2, p. 164-171, 2008.

ROSKIN, J. et al. Do dune sands redden with age? The case of the northwestern Negev dunefield, Israel. Aeolian Research, v. 5, p. 63-75, 2012.

ROSSEL, R. A. V. et al. Colour space models for soil science. Geoderma, v. 133, n. 3-4, p. 320-337, 2006.

ROSSEL, R. A. V.; FOUAD, Y.; WALTER, C. Using a digital camera to measure soil organic carbon and iron contents. Biosystems Engineering, v. 100, n. 2, p. 149-159, 2008.

RUGGIERO, P. G. C. et al. Relação entre solo, vegetação e topografia em área de cerrado (Parque Estadual de Vassununga, SP): como se expressa em mapeamentos? Acta Botanica Brasilica, v. 20, n. 2, p. 383-394, 2006.

SANTANA, O. A.; CUNIAT, G.; IMAÑAENCINAS, J. Contribuição da vegetação rasteira na evapotranspiração total em diferentes ecossistemas do bioma Cerrado, Distrito Federal. Ciência Florestal, v. 20, n. 2; p. 269-280, 2010.

SANTANA, O. A.; IMAÑA-ENCINAS, J. Leaf Area Index and Canopy Openness estimation using high spatial resolution image QuickBird. Revista Caatinga, v. 24, p. 59-66, 2011.

SANTANA, O. A. et al. Produção da serapilheira em Floresta de Galeria e Floresta Mesofítica na Dolina da Garapa, Distrito Federal, Brasil. Cerne, v. 16, n. 4, p. 585-596, 2010.

SCHWERTMANN, U. Relations between iron oxides, soil color, and soil formation. In: J.M. Bigham and E.J. Ciolkosz. SSSA. (Ed.). Soil Color. Madison. 1993. p. 57-66. (Special Publication, 31). TEREFE, $T$. et al. Influence of heating on various properties of six Mediterranean soils. A laboratory study. Geoderma, v. 143, n. 3-4, p. 273280, 2008.

TORRENT, J.; BARRÒN, V. Laboratory measurement of soil color: theory and practice. In: J.M. Bigham and E.J. Ciolkosz. SSSA. (Ed.). Soil Color. Madison. 1993. p. 21-33. (Special Publication, 31).

VALE, A. T.; FELFILI, J. M. Dry biomass distribution in a cerrado sensu stricto site in Brazil central. Revista Árvore, v. 29, n. 5, p. 661-669, 2005.

VIANNA, A. L. M. et al. Tamanho ótimo de parcela para estimativa de estoque de carbono da parte aérea lenhosa de fitofisionomias florestais na Amazônia Oriental. Floresta, v. 40, n. 2, p. 447456, 2010.

VIEIRA, S. A. et al. Estimation of biomass and carbon stocks: the case of the Atlantic Forest. Biota Neotropica, v. 8, n.2, p. 21-29. 2008.

WATERWORTH, R. M.; RICHARDS, G. P. ImplementingAustralian forestmanagementpractices into a full carbon accounting model. Forest Ecology and Management, v. 255, n. 7, p. 2434-2443, 2008. ZAR, J. H. Biostatistical analysis. 5th Ed. New Jersey: Prentice Hall, 2010. 944 p. 